\title{
REPENSANDO LA ENSEÑANZA \\ DE L2 EN PROGRAMAS DE ESTUDIO EN EL EXTRANJERO (PREE): CONSIDERACIONES A PARTIR DE UNOS CURSOS DE ESPAÑOL UNIVERSITARIOS
}

\author{
MARÍA VICTORIA LÓPEZ PÉREZ \\ Universidad Pública de Navarra, España
}

\begin{abstract}
Resumen. Aunque algunas universidades españolas cuentan con una extensa experiencia en la recepción de estudiantes extranjeros en programas de EL2 (PREE), otras, generalmente de reciente creación, están empezando a prestarles atención, entre otros motivos, como parte de su apuesta por la internacionalización. Es el caso de la Universidad Pública de Navarra, España, que, en la última década, viene ofreciendo cuatro asignaturas de español L2 a más de 150 estudiantes internacionales que las cursan anualmente como parte de su programa de estudios en la universidad de acogida. Actualmente se está llevando a cabo una investigación cuyas conclusiones servirán de referencia para revisar los objetivos de aprendizaje y los programas de las mencionadas asignaturas, o para la elaboración de otros destinados a asignaturas EL2 de nueva creación que se ajusten al perfil, a las necesidades, a los intereses y a las expectativas de los estudiantes internacionales a los que van dirigidas. La investigación en curso, de tipo cuantitativo, tiene como muestra un centenar de estudiantes de los cursos 2017-18 y 2018-19, y analiza el perfil sociolingüístico de los estudiantes, sus motivaciones para aprender español y sus actitudes hacia los distintos elementos que comprenden el contexto pedagógico de inmersión en que se desarrollan estas asignaturas. Los temas incluidos en la investigación y resultados parciales de esta nos sirven en este trabajo para reflexionar sobre aspectos pedagógicos y de investigación relacionados con los PREE.
\end{abstract}

Palabras clave: español como lengua segunda, programas de estudio en el extranjero, estudiantes universitarios, programación, análisis de necesidades, factores afectivos

\section{INTRODUCCIÓN}

En este trabajo se ofrece una reflexión sobre los Programas de estudio en el extranjero (PREE) a partir de experiencias de aula y de investigación de uno de estos cursos de español como lengua segunda (EL2) ofrecidos por una universidad 
española, la Universidad Pública de Navarra (UPNA). Desde su Facultad de Humanidades, e incardinadas en unos de sus estudios de grado, esta universidad ofrece cuatro asignaturas de EL2, dos en el primer semestre y dos en el segundo, cuya finalidad es mejorar la competencia comunicativa de los estudiantes extranjeros de intercambio, de grado y posgrado. Cada una de estas asignaturas, a las que nos referiremos también como cursos, tiene 6 créditos y pueden ser elegidas por el alumnado de diferentes carreras universitarias para completar su programa académico en la universidad de acogida. Dos de estas asignaturas, Habilidades comunicativas en español para extranjeros I y II, se llevan ofreciendo desde hace ya una década. Las asignaturas de Lengua, cultura y literatura I y II son de reciente implantación. En las primeras se imparte español con fines generales, en los niveles A2 y $\mathrm{B} 1$ del MCER; las segundas, plantean un aprendizaje de lengua a través de contenidos culturales, en los niveles B1 y B2 del MCER. La oferta de todas ellas obedece a un plan estratégico de internacionalización por parte de la UPNA y no son estas las únicas asignaturas de EL2 que oferta la universidad. Junto a otros idiomas, los estudiantes internacionales pueden además estudiar español como lengua segunda en el Centro Superior de Idiomas, institución también dependiente de la universidad, pero en este caso, los cursos nos son gratuitos. Desde Relaciones Internacionales y Cooperación, dependiente del Vicerrectorado de Internacionalización de la UPNA, se acompaña la aclimatación de los estudiantes extranjeros a la universidad con actuaciones de acogida como el programa Buddy. La finalidad de este programa es apoyar a este alumnado a integrarse socialmente y académicamente en la universidad y en la ciudad. En él participan, de forma voluntaria, estudiantes de la universidad que tienen o van a tener una experiencia de intercambio similar en el extranjero, y cualquier estudiante a partir de segundos cursos. A los estudiantes participantes se les reconoce el trabajo en el programa Buddy con 1 ECTS.

Las cuatro asignaturas citadas en el párrafo anterior se encuentran en un proceso de revisión con el fin de introducir intervenciones pedagógicas que mejoren las que actualmente se llevan a cabo. Para esta revisión se va a tomar como referencia las conclusiones de una investigación iniciada en el curso académico 2017-2018, sobre una muestra de un centenar de estudiantes, que tiene como objetivo reunir información que permita trazar un perfil sociolingüístico de los estudiantes que cursan estas asignaturas, averiguar sus necesidades comunicativas en español, y conocer su motivación y sus actitudes respecto al aprendizaje de esta lengua. Así pues, los resultados de la investigación guiarán las modificaciones que se introduzcan en la programación de las asignaturas para adecuarlas a las características, a los intereses, a las necesidades y a las expectativas del alumnado al que van dirigidas. También servirán para idear un sistema que ponga las cuatro asignaturas en relación para que los estudiantes puedan cursar no solo una, sino varias de ellas durante su estancia en la universidad, y conseguir con ello progresar en el dominio del español. Además de orientar la docencia de las asignaturas en ediciones posteriores de las asignaturas, esta información servirá de base para la creación otras nuevas de EL2 que completen la oferta. 
Las conclusiones de los distintos temas abordados en la investigación pueden contribuir a la investigación en los campos Didáctica de EL2 y en el de Adquisición de segundas lenguas. Estos temas, así como los resultados parciales de dicha investigación, nos sirven en este trabajo de hilo conductor para reflexionar sobre aspectos atingentes a los programas de estudio en el extranjero.

\section{LA INVESTIGACIÓN SOBRE FACTORES QUE INDICEN EL APRENDIZAJE EN DOS ASIGNATURAS DE EL2}

\subsection{TEMAS Y OBJETIVOS DE LA INVESTIGACIÓN}

Como recuerda Moreno Fernández (2018: 288): 'los factores que operan en cualquier proceso de adquisición de lenguas son variados y la cantidad y complejidad de las dimensiones que cada uno de ellos encierra es bastante importante'. Exponemos aquí por esclarecedor el resumen que de ellos hace Ezpí y Azurmendi (1996: 64):

Factores relacionados con el aprendiz:

Individuales: inteligencia, aptitud lingüística, estrategias de aprendizaje, personalidad.

Socio-lingüísticos y demográficos: edad, género, nivel educativo, nivel sociocultural y socioeconómico, lengua nativa, lenguas que domina, nivel de dominio de la lengua meta.

Psicosociales o socio-afectivos: identidad social, la motivación hacia el aprendizaje de la L2, las actitudes respecto a la L2 y a la comunidad lingüística/cultural de la L2.

Factores pedagógicos: metodología, recursos didácticos, profesorado y contexto de aprendizaje.

Factores contextuales sociales o sociopolíticos que refieren al estatus que las distintas lenguas ocupan en la sociedad.

Esta clasificación no es ni definitiva ni exhaustiva. Por ejemplo, no contempla como factor socio-lingüístico las culturas educativas de las que provienen los aprendices, o entre los individuales, los estilos de aprendizaje, pero se tuvo en cuenta a la hora de concretar los temas de nuestra investigación. Esta se fija en los factores relacionados con el aprendiz: socio-lingüísticos y demográficos, socioafectivos, y en factores pedagógicos, como puede observarse en los objetivos que se marca:

1. Obtener un perfil sociolingüístico de los estudiantes.

2. Obtener el perfil motivador y actitudinal del grupo de estudio.

3. Identificar correlaciones entre factores individuales (género, nivel de español, número de lenguas que hablan, contacto con el mundo hispano, distancia de la lengua nativa del estudiante con la lengua española), y las variables afectivas estudiadas. 
4. Identificar factores que interactúan en el proceso de enseñanzaaprendizaje y que influyen tanto en el éxito del aprendizaje de la lengua meta como en la motivación para aprenderla (motivación, actitudes, rendimiento lingüístico).

Relacionados con los objetivos están las preguntas de investigación a las que tratará de responder la investigación:

¿Qué perfil general presentan los estudiantes que eligen estas asignaturas (nacionalidad, género, lenguas que hablan, contacto con el español, nivel de español)?

¿Cuáles son los motivos que conducen a los estudiantes a elegirlas? ¿Cómo valoran los elementos de la situación de aprendizaje: profesorado, curso, grupo, recursos didácticos y programáticos?

¿Qué tipo de contenidos socioculturales y en qué ámbito (España/ Latinoamérica) les interesan?

¿Se aprecian influencias de los factores individuales sobre las variables de tipo afectivo?

¿Tienen las motivaciones y las actitudes de los estudiantes influencia en su rendimiento lingüístico?

Como puede apreciarse, se trata de una investigación ambiciosa, con una muestra representativa, un centenar de estudiantes, y numerosas preguntas, cuyos datos recogidos están siendo analizados utilizando medios estadísticos. Las conclusiones de las preguntas de investigación que requieren de un análisis descriptivo correlacional serán próximamente publicadas para ponerlas a disposición de la comunidad investigadora. En este trabajo se incluyen resultados parciales de la investigación obtenidos del análisis de 44 sujetos de la muestra, y solo de algunas cuestiones que nos sirven para ejemplificar los aspectos sobre los que vamos reflexionar y no requieren un complejo estudio estadístico.

\subsection{METODOLOGÍA: PARTICIPANTES Y CUESTIONARIO}

La investigación se ha realizado a partir de las respuestas de 97 estudiantes de ediciones de las asignaturas Habilidades comunicativas I y II, en los años académicos 2017-18 y 2018-19. La recogida de datos se llevó a cabo en un cuestionario en línea confeccionado con google forms, en español y en inglés, que se pasó en clase el último día de los cursos. Con ligeras modificaciones para adaptarlo al contexto de investigación propio, se trata del cuestionario de Minera Reyna (2010) Motivación y actitudes en el aprendizaje del español como lengua extranjera en Alemania (MAALE) y contiene los siguientes apartados:

0 . Datos socio-lingüísticos

I. Tipos de motivación (integradora, instrumental, intrínseca, extrínseca)

II. Tipo de motivación dominante

III. Grado de motivación 
IV. Actitudes

- Actitud hacia sí mismo

- Actitud hacia la situación de aprendizaje (profesorado, asignatura, grupo, elementos del entorno didáctico)

- Actitud general hacia las lenguas extranjeras

- Actitud general hacia lo español y los españoles

- Actitud general hacia los países de habla hispana y sus gentes

V. Temas y ámbitos de preferencia

Las preguntas del cuestionario son cerradas, con escalas Likert y de diferencial semántico en sus respuestas, excepto la última en la que los estudiantes dejan constancia de los temas no tratados en el curso y que les gustaría trabajar en clase. La investigación, por tanto, va a ofrecer un análisis de datos cuantitativo, para lo que se va a utilizar la herramienta estadística " $R$ ".

\section{REFLEXIÓN SOBRE DATOS SOCIOLINGÜÍSTICOS Y DEMOGRÁFICOS}

En el análisis de la muestra de 44 estudiantes, se observan tres datos: la abrumadora mayoría de mujeres (70\%), los distintos niveles de español de los estudiantes: A1 (22\%), A2 (9\%), B1 (22\%), B2 (34\%) C1 (13\%) y la cantidad de nacionalidades distintas dentro de un mismo grupo, diecisiete. Los estudiantes preceden de los cinco continentes, reflejo del alcance de los convenios internacionales que desarrolla la universidad. El dato de la edad no es significativo, puesto que la mayoría ronda los 20-21 años.

Respecto a los niveles, las asignaturas estudiadas se imparten en dos niveles A1-A2 y B1, aunque se sigue el principio de admitir a los todos estudiantes que las quieran cursar independientemente del nivel que tengan de español y, eso explica que haya un pequeño porcentaje que, a pesar de tener un nivel superior (C1), acudan a las clases. Por otra parte, los estudiantes han podido responder cuando se les preguntó por su nivel de español, el nivel en el que fueron ubicados o en el adquirido al final de curso. Recordemos que el cuestionario se pasó al final de este. Esta diversidad de nacionalidades, idiomas, y niveles de lengua puede considerarse una dificultad frente a cursos PREE en los que los grupos de estudiantes proceden de un mismo país o comparten un único idioma a nivel nativo. Sin embargo, esta heterogeneidad puede considerarse como un valor positivo y servir al desarrollo de la competencia intercultural del alumnado por la posibilidad de compartir con estudiantes de otras culturas un mismo espacio de aprendizaje. Como Moreno Fernández (2018: 292) indica: 'la comunicación intercultural, en relación con la $A S L$, se produce cuando al aprendiz está inserto en una cultura y un contexto lingüístico diferentes a los suyos o en contacto con hablantes o aprendices procedentes de otras culturas'. La forma de hacer frente a esta situación de diversidad por parte de los programadores y profesores de las asignaturas es adoptar una metodología ecléctica, que incluya distintos 
enfoques comunicativos para que dé encaje a las distintas culturas educativas de las que proceden los estudiantes, procurar tiempos en las sesiones en los que se puedan atender conjuntamente a estudiantes que comparten la misma lengua y los mismos problemas frente al aprendizaje del español, por ejemplo, problemas de pronunciación en los estudiantes chinos, e idear sistemas de evaluación flexibles, como el que mostramos en el Anejo 1. A todo ello contribuye también la elaboración de proyectos en grupo bien relacionados con la cultura hispánica o española o bien con los estudios de grado que cursan, en los que se potencia el empleo de las TIC y que son expuestos ante los demás estudiantes. De este modo, se potencia, de modo positivo, la autonomía en su propio aprendizaje, y la adquisición y asimilación de valores culturales. Por último, no se deben descuidar los aspectos emocionales, y una forma de atenderlos es crear un clima de aula en que los aprendices se sientan incluidos y sus personas y culturas respetadas.

\section{REFLEXIÓN SOBRE FACTORES AFECTIVOS}

Los factores socio-afectivos como la motivación, las actitudes y las creencias, son hoy también reconocidos por la comunidad investigadora, junto a los factores de tipo cognitivo, como responsables del aprendizaje. Los factores afectivos, como indica Dornyei (2005, citado en Tovar y Cando, 2019: 2) deben ser evaluados frecuentemente por profesores y autoridades académicas en las programaciones de lenguas: 'Language instructors and school administrators, then should frequently check and revise not only the educational goals and beliefs but explore learners' interest, effort, desire and attitude towards learning'. Para Gadner (1985: 10) el concepto motivación hace referencia 'to the extent to which the individual works or strives to learn the language because of a desire to do so and the satisfaction experience in this activity'. Según este mismo autor (Gadner, 1982), la motivación activará el esfuerzo (el tiempo que emplea estudiando y el interés que le pone el estudiante), el deseo (el anhelo de dominar la lengua meta), y el afecto (las reacciones emocionales del aprendizaje frente al estudio). Por la relación de la motivación con el rendimiento académico y éste con la eficacia de la enseñanza y los niveles de aprendizaje (Spolsky, 1989), se consideró de interés averiguar en nuestra investigación los tipos de motivación de nuestros estudiantes, la motivación dominante global del grupo y el grado de motivación. Sin entrar en este momento en disquisiciones conceptuales sobre las categorizaciones de los tipos de motivación, son dos los tipos de motivación que se han planteado en la investigación: extrínseca e intrínseca. La primera engloba las motivaciones: integrativa, instrumental y resultativa en la taxonomía de Ellis (1997: 75-76). En la muestra parcial se ha encontrado que la motivación predominante es la extrínseca, pero la intrínseca también está presente en gran medida. Entre los motivos de naturaleza intrínseca los aprendices señalan el gusto por aprender cosas nuevas. Las motivaciones instrumentales que se han encontrado en un mayor número de respuestas son: aprendizaje del español para viajar, aprendizaje para facilitar la relación con personas hispanohablantes y aprendizaje para ser más 
instruido. Llama la atención que los motivos para aprender español profesiones o académicos no han sido los más señalados. Estos resultados vienen a coincidir con los de un estudio realizado por Espí y Azurmendi (1996) realizado en 1992-93 sobre una muestra de 69 estudiantes de español de edades semejantes a los nuestros de un PREE en España. Una conclusión que sacamos de ello es que a pesar de que el español ha ganado fuerza internacional en lo profesional y lo científico desde los años en que las autoras anteriores realizaron el estudio, casi treinta años después, los estudiantes no aprecian la utilidad de nuestro idioma en esos ámbitos. El pronóstico que hacían entonces Espí y Azurmendi (1996: 70) de que la presencia del español en el mundo laboral y de los negocios a nivel internacional aumentaría, y que esto llevaría a los aprendices de español a apreciar la utilidad del español en estos campos no se ha cumplido.

La orientación didáctica de esta disquisición acerca de la motivación en el marco de los PREE nos lleva a incluir aquí lo que Lorenzo Berguillos (2018: 319-322) llama Macroestrategias motivacionales. Se trata de un listado de buenas prácticas por parte de los profesores de segundas lenguas, refrendadas por la investigación en enseñanza de lenguas, para conseguir aumentar la motivación de los estudiantes:

- L2 Transmitir un ejemplo de compromiso con la disciplina y asumir los objetivos de enseñanza por parte del profesor

- Crear una atmósfera relajada en el aula

- Presentar actividades de una forma ordenada, con objetivos definidos y graduados a los niveles de los alumnos.

- Hacer las clases interesantes

- Promover la autonomía en el aprendizaje

- Familiarizar a los alumnos con la cultura de la lengua 2

Por su papel también como atenuadores o favorecedores del aprendizaje, se ha creído procedente en la investigación analizar las actitudes de los estudiantes hacia su autoconfianza como aprendices de lenguas segundas, hacia los países, personas y culturas del ámbito hispánico y hacia distintos elementos de la situación de aprendizaje. De acuerdo con Ajzen, (2005: 3), actitud es 'a disposition to respond facovable or unfavorable to an object, person, institution, or event'. Ejemplos de actitudes el campo de aprendizaje de lenguas pueden ser impresiones positivas o negativas sobre aspectos como la dificultad o simplicidad de una lengua, la facilidad o dificultad del aprendizaje, las preferencias de aprendizaje o uso de la lengua, el grado de importancia de la lengua, su agrado estético, incluso la consideración que los aprendices tienen respecto a los hablantes de esa lengua (Baker, 1992). Las actitudes son evaluables y pueden crearse o modificarse a través de la experiencia de contacto con la cultura, la lengua o los hablantes de esa lengua (Ezpí y Azurmendi, 1996). En las actitudes influyen distintas variables como la edad, el género, el contexto cultural, el entorno lingüístico, el contexto educativo y la aptitud lingüística del aprendiz (Baker, 1992). Nuestra 
investigación tiene previsto estudiar las correlaciones entre los valores afectivos motivación y las actitudes, con el género y el nivel de español.

\section{REFLEXIÓN SOBRE EL CONTEXTO PEDAGÓGICO}

Entre los factores pedagógicos suelen distinguirse la metodología, los recursos didácticos, el profesorado y el contexto de aprendizaje. Los primeros han sido unos de los más estudiados en la Didáctica de la lengua y no podían faltar en nuestra investigación que incluye entre sus fines mejorar los programas de las asignaturas y poner en evidencia a partir de las valoraciones de estudiantes áreas de mejora en la programación, en las actuaciones del profesorado y en las condiciones ambientales de clases. Así, se incluyen en ella preguntas en las que los estudiantes valoran al profesorado, a los compañeros/as, la asignatura, los recursos didácticos y las condiciones ambientales del aula. Como comentario general se aprecia que los estudiantes dan su aprobado a los distintos elementos evaluados, con una suma de los valores muy bueno y bueno del $60 \%$. Se encontró que obtienen una valoración más baja en el sistema de evaluación (60\%) y en la plataforma virtual (59\%). Respecto al sistema de evaluación llevado en práctica en las asignaturas de las que nos ocupamos, evalúa tanto procesos como resultados, y se plantea de forma flexible ya que se compone distintas tareas que deben ser realizadas en colaboración o de forma individual, y que, además, tienen la particularidad de que no todas son obligatorias (véase Anejo 1). Los estudiantes pueden dejar de realizar algunas de estas tareas, pero esta decisión les impide alcanzar las máximas calificaciones ya que pierden el porcentaje que esa tarea representa en la nota final. Tras la disconformidad por parte del alumnado con el sistema de evaluación detectada en la investigación, se introdujo un examen que mide los resultados de aprendizaje de las destrezas comprensión lectora, comprensión auditiva y competencia gramatical y léxica.

La investigación también indaga sobre la acogida que los estudiantes hacen de los temas culturales incluidos en la programación e insta a proponer otros de su interés. Los resultados han arrojado el dato de que el 70\% de los estudiantes prefieren que en los cursos se traten temas no solo de España, sino también de países hispanoamericanos, contradiciendo la creencia de los programadores en el sentido de que porque los cursos tienen lugar en España, los estudiantes preferirían de forma mayoritaria que los temas se centrasen en este país.

Hay un elemento más que conforma el contexto pedagógico en los PREE en relación con los cursos de lenguas segundas que tienen lugar en contextos formales de aprendizaje en comunidades lingüísticas en las que lengua meta no está presente: el entorno social y cultural. Este entorno cobra especial protagonismo porque puede convertirse en un potente factor favorecedor del aprendizaje. Como señalan Lafford y Isabelli (2018: 505), el contexto pedagógico 'se amplía con actividades de acompañamiento promovidas tanto por los organizadores y como con las propias experiencias de estudiantes cuando interactúan con las comunidades de la lengua meta'. Las diferentes 
subcompetencias que integran la competencia comunicativa salen reforzadas por ello, pero sin duda es la competencia sociocultural, según el modelo de CelceMurcia, Dönyei y Thurrell (1995), o la intercultural según el modelo de Byran y Fleming (2001), las que salen más beneficiadas. Sobre esta última se encuentran estudios como el de Alfonzo, Cáceres y Santana (2017), que analiza los perfiles interculturales de estudiantes erasmus en una universidad española. Ahora bien, en qué grado, esta especificidad del contexto de inmersión de los PREE potencia las citadas competencias dependerá de la calidad y cantidad de las interacciones entre estos y los nativos, y aquí entran en juego factores individuales y sociales que están presentes en dichas interacciones (Magnan y Lafford, 2012). A pesar de la importancia del contexto pedagógico en los $P R E E$, es fácilmente constatable, y así lo señalan también Lafford e Isabelli (2018: 505) y LcLeod y Wainwright (2009:66), que hay una falta de trabajos que analicen con profundidad la naturaleza de este contexto para entender la experiencia total del estudiante, en cuanto al desarrollo de su competencia comunicativa y a los efectos que el contacto con otras culturas le produce a nivel existencial. Pueden contribuir a los estudios anteriormente mencionados las conclusiones a las que se ha llegado tras estudiar los datos de la muestra total de nuestra investigación sobre la incidencia en su motivación del contacto con nativos de los estudiantes antes y durante el curso PREE. Se ha encontrado que los contactos con hispanohablantes previos al $P R E E$ no influyen en la motivación de los aprendices, pero que, por el contrario, el empleo del español fuera de clase durante el PREE sí contribuye a aumentar su motivación por el aprendizaje (López Pérez, 2019).

\section{CONCLUSIONES}

En este trabajo nos propusimos reflexionar sobre cuestiones relacionadas con la enseñanza-aprendizaje de segundas lenguas en programas de estudio en el extranjero, y para ello nos han servido de guía los temas incluidos en una investigación en curso sobre uno de estos programas de español/L2 dirigidos a estudiantes universitarios de intercambio en una universidad española.

La primera reflexión no por obvia, es menos importante. La elaboración de programas enseñanza-aprendizaje de lenguas deben tener en cuenta el análisis de las necesidades de los estudiantes. Estos pueden servir de punto de partida o, como es nuestro caso, realizarse en la trayectoria ya iniciada de los cursos para evaluar y corregir aspectos curriculares que no se ajusten a las necesidades, a los intereses o a las expectativas de los aprendices. En casos de grupos de estudiantes heterogéneos en cuanto a nacionalidades, idiomas y culturas educativas, este análisis es aún más pertinente. En nuestra investigación hemos hallado contradicciones entre algunos elementos del programa y la percepción o valoración que los estudiantes tenían de ellos. Es, por ejemplo, el caso del libro de texto, que fue cambiado por otro porque el profesorado creyó erróneamente que no satisfacía a los estudiantes, o del sistema de evaluación, que después de conocerse por los resultados de la investigación, se modificó para incluir un 
examen que los estudiantes demandaban. Relacionado con lo metodológico y las prácticas docentes en los $P R E E$, hay un gran espacio para la investigación, como indican Lafford e Isabelli (2018: 506). Serían de interés estudios como los López Pérez y Bueno Alastuey (2015) que explore la aportación que las TIC hacen a los aprendizajes en este tipo de cursos de inmersión.

En los PREE, se ha de atender asimismo a factores de tipo afectivo por su demostrada relación con el rendimiento lingüístico. Es necesario contar con un conocimiento fidedigno, no apriorístico, de factores como las creencias, la motivación y las actitudes que los estudiantes tienen hacia el aprendizaje de la lengua meta. Se encontró que el tipo de motivación que predomina en el conjunto de estudiantes investigado es la extrínseca, y que lo que les mueve a la mayoría a estudiar español no son razones de tipo profesional, sino social y personal. Los estudiantes manifiestan que estudian español para relacionarse con personas de habla hispana y para viajar. Respecto a las actitudes, las positivas como la sensibilidad cultural de los aprendices, actúan como facilitadores del aprendizaje. En el caso de las sesgadas o las negativas, pueden obstaculizar la progresión de este, así como propiciar sinergias en el grupo de aprendizaje que interfieran en la cohesión del mismo. Detectando este último tipo de actitudes, como se pretende en la investigación iniciada, se puede tratar de influir en ellas con distintas acciones, desde metodológicas hasta extracurriculares, como propiciar experiencias de contacto con la cultura meta.

En los PREE, el contexto pedagógico se amplía con los elementos humanos y culturales que pertenecen a la lengua meta. La interacción de los estudiantes con estos elementos favorece los aprendizajes lingüísticos, sobre todo en la competencia sociocultural y el desarrollo de su conciencia intercultural. Ahora bien, como recuerdan Lafford e Isabelli (2018: 506), 'los aprendices necesitan desempeñar un papel activo e interactuar en redes sociales profundas de la comunidad anfitriona' para aprovechar las ventajas que el contexto les ofrece. Ahora bien, debe ser otra función de los responsables de los programas de cursos PREE planificar acciones que faciliten el contacto de los aprendices con el medio en el que están inmersos. El programa Buddy desarrollado por la UPNA es un buen ejemplo de este tipo de acciones. Así mismo, se deben enseñar a los estudiantes estrategias para el aprendizaje cultural y lingüístico. Manuales con este tipo de estrategias como el de Paige et al. (2014) elaborados por profesores de universidades norteamericanas específicamente para PREE se echan de menos en los programas organizados por instituciones europeas. Por otra parte, para promover el desarrollo de la conciencia intercultural, se pueden incluir en la programación tareas que propicien una reflexión crítica sobre el aprendizaje de la lengua meta y la experiencia social y cultural de los estudiantes, como la confección de un portafolio. Falta también investigación que arroje luz sobre en qué medida los elementos del contexto pedagógico de inmersión influencian el aprendizaje de una segunda lengua. 
Por último, la investigación a la que nos hemos referido en este trabajo deja muchas parcelas sin cubrir que contribuirían también a una más completa reflexión sobre la enseñanza de lenguas segundas en los PREE. Por ejemplo, en los últimos años se ha observado que entre los estudiantes internacionales los hay que tienen el español como lengua de herencia. El cuestionario no los identifica y somos conscientes de que estos estudiantes exigen un tratamiento pedagógico diferenciado.

\section{REFERENCIAS BIBLIOGRÁFICAS}

Ajzen, I. (2005) Attitudes, Personality and Behavior. New York: Open University Press.

Alfonzo de Tovar, I. C., Cáceres Lorenzo, M. T. y Santana Alvarado, Y. (2017) Erasmus+ Student Profile in the Development of Intercultural Competence: A Case Study. RAEL, 16 (1): 103-117.

Baker, C. (1992) Attitudes and Language. Clevedon. Philadelphia, Adelaide: Multilingual Matters Ltd.

Byram, M. y Fleming, M. (2001) Perspectivas interculturales en el aprendizaje de idiomas. Enfoques a través del teatro y la etnografía. Madrid: Cambridge University Press.

Celce-Murcia, M., Dönyei, Z. y Thurrell, S. (1995) A pedagogically motivated model with context specificatios. Issues in Applied Linguistics, 6: 5-35.

Ellis, R. (1997) Second Language Acquisition. Oxford: Oxford University Press.

Espí Guzmán, M. J. y Azurmendi Ayerbe, M. J. (1996) Motivación, actitudes y aprendizaje en el español como lengua extranjera. RESLA, 11: 63-76.

Gardner, R. C. (1982) Language attitudes and language learning. In E. Bouchard Ryan and H. Giles (eds.) Attitudes Towards Language Variation (pp. 132-147). London: Edward Arnold.

Gardner, R. C. (1985) Social psychology and language earning: The role of attitudes and motivation. London: Edward Arnold.

Lafford, B. A. e Isabelli, C. A. (2018) Programas de estudio en el extranjero. En J. Muñoz-Basols, E. Gironzetti y M. Lacorte (eds.) The Routledge Handbook of Spanish Language Teaching: metodologías, contextos y recursos para la enseñanza del español L2 (pp. 505-517). London and New York: Routledge.

LcLeod, M. y Wainwright, Ph. (2009) Researching the Study Abroad Experience. Journal of Studies in International Education, 13 (1): 66-71.

López Pérez, M. V. (2019) Incidencia del contacto con nativos en la motivación de aprendices de ELE en un PREE. En $30^{\circ}$ Congreso Internacional de ASELE 2019. Internacionalización y enseñanza del español como LE/L2: plurilingüismo y comunicación intercultural (p. 105). Disponible en https://objsrv01.eventqualia.com/evt4-media/documents/Version_final_ Libro_de_resumenes_ASELE_2019-2.pdf [Consultado el 20 de septiembre de 2019].

López Pérez, M. V. y Bueno Alastuey, M. C. (2015) Una experiencia de enseñanza combinada en un curso universitario de español/L2. Percepciones de los estudiantes sobre el efecto de las TIC en sus aprendizajes. RESLA, 28 (1): 214-233.

Lorenzo Berquillos, F. J. (2016) La motivación y el aprendizaje de una L2/LE. En J. Sánchez Lobato e I. Santos Gargallo (eds.) Vademécum para la formación de profesores. Enseñar español como segunda lengua (L2)/lengua extranjera (LE), tomo I, (pp. 305-328). Madrid: SGEL.

Magnan, S. y Lafford, I. (2012) Learning through Immersion during Study Abroad. En S. M. Gass y A. Mackey (eds.) The Routledge Handbook of Second language Acquisition (pp. 525-540). London, New York: Routlegde. 
Minera Reyna, L. E. (2010) El cuestionario MAALE, técnica para la recolección de datos de las variables afectivas motivación y actitudes en el aprendizaje de una lengua extranjera. REDele Revista electrónica de didáctica/ español lengua extranjera, 19. Disponible en https://dialnet. unirioja.es/servlet/articulo?codigo=3267684 [Consultado el 5 de marzo de 2019].

Moreno Fernández, F. (2016) El contexto social y el aprendizaje de una L2/LE. En J. Sánchez Lobato e I. Santos Gargallo (eds.) Vademécum para la formación de profesores. Enseñar español como segunda lengua (L2)/lengua extranjera (LE), tomo I, (pp. 208-304). Madrid: SGEL.

Paige, R. M., Cohen, A. D., Kappler, B., Chi, J. C. y Lassegard, J. P. (2014) Maximizing Study Abroad: A Students' Guide to Strategies for Language and Culture Learning and Use. Center for Advanced Research on Languae Acquisition. 2nd ed. Minneapolis: University of Minessota.

Spolsky, B. (1989) Conditions for Second Language Learning. Oxford: Oxford University Press.

Tovar, R. y Cando, F. S. (2019) Motivation, attitude and anxiety as determiners of EFL language learning. En 2nd International Congress Fostering active learning in EFL classroom: connecting teaching learning and research. (pp. 1-7). Disponible en https://congresoidiomas.uta.edu. ec/lectures.html [Consultado el 2 de septiembre de 2019].

\title{
RETHINKING THE TEACHING OF L2 IN STUDY ABROAD (SA) PROGRAMS: CONSIDERATIONS FROM UNIVERSITY COURSES OF SPANISH
}

\begin{abstract}
Although some Spanish universities have extensive experience in receiving students in Spanish as a Second Language SA programs, others, generally of recent creation, are beginning to take notice among other reasons, as part of their commitment, to internationalization. This is the case of the Public University of Navarra, Spain, which, in the last decade has been offering four L2 Spanish courses to more than 150 international students who attend them annually as part of their study program at the host university. Currently an investigation is being carried out the conclusions of which will serve as a reference to review the learning objectives and the programs of the subjects afore mentioned, or others will be developed for newly created EL2 subjects that are adequate for the profile, needs, interests and the expectations of the international students to whom they are addressed. The ongoing research, of quantitative type, has as a sample a hundred students of the 2017-18 and 2018-19 courses, and analyzes the sociolinguistic profile of the students, their motivations to learn Spanish and their attitudes towards the different elements that comprise the pedagogical immersion context in which these subjects are developed. The topics included in the research and its partial results serve us in this work to reflect on pedagogical and research aspects related to the SA programs.
\end{abstract}

Key words: Spanish as a second language, study abroad programs, university students, language teaching, needs analysis, affective factors 


\section{ANEJO 1}

Sistema de evaluación de la asignatura

\begin{tabular}{|l|c|c|}
\hline \multicolumn{1}{|c|}{ CRITERIOS DE EVALUACIÓN } & $\begin{array}{c}\text { INSTRUMENTOS DE EVALUACIÓN / } \\
\text { TIPO DE TAREA }\end{array}$ & $\begin{array}{c}\% \\
\text { NOTA }\end{array}$ \\
\hline $\begin{array}{l}\text { Aprovechamiento de los recursos } \\
\text { educativos a través de la presencia activa } \\
\text { y cooperativa del estudiante en clase. }\end{array}$ & $\begin{array}{c}\text { REGISTRO DIARIO de la } \\
\text { asistencia y de la participación por } \\
\text { parte del docente. } \\
\text { Obligatoria la asistencia a un 50\% } \\
\text { de las sesiones impartidas. }\end{array}$ & $20 \%$ \\
\hline $\begin{array}{l}\text { Grado alcanzado en el desarrollo de las } \\
\text { destrezas: comprensión oral y lectora, } \\
\text { competencias gramatical y léxica. }\end{array}$ & $\begin{array}{c}\text { EXAMEN ESCRITO } \\
\text { Tarea individual, obligatoria. }\end{array}$ & $25 \%$ \\
\hline $\begin{array}{l}\text { Desarrollo demostrado en comprensión } \\
\text { lectora, en expresión oral y en } \\
\text { competencia intercultural. }\end{array}$ & $\begin{array}{c}\text { PARTICIPACIÓN EN } \\
\text { TERTULIA LITERARIA }\end{array}$ & $10 \%$ \\
\hline $\begin{array}{l}\text { Desarrollo demostrado en comprensión } \\
\text { lectora, en expresión escrita y en } \\
\text { competencia digital. }\end{array}$ & $\begin{array}{c}\text { INFORME obligatoria. } \\
\text { Tarea por parejas, no } \\
\text { obligatoria. }\end{array}$ & $10 \%$ \\
\hline $\begin{array}{l}\text { Reflexión y control del estudiante sobre } \\
\text { su propio proceso de aprendizaje. }\end{array}$ & $\begin{array}{c}\text { PORTAFOLIO } \\
\text { Tarea individual, no obligatoria. }\end{array}$ & $15 \%$ \\
\hline $\begin{array}{l}\text { Desarrollo demostrado en competencia } \\
\text { discursiva. }\end{array}$ & $\begin{array}{c}\text { EXPOSICIÓN ORAL } \\
\text { Tarea por parejas, obligatoria. }\end{array}$ & $20 \%$ \\
\hline
\end{tabular}

María Victoria López Pérez (Dra., Prof.a Contratada Doctora) imparte docencia en la Universidad Pública de Navarra. Sus campos de investigación son Factores socio-afectivos en la enseñanza-aprendizaje de lenguas y el Español como lengua académica en niveles preuniversitarios. Correo electrónico: victoria.lopez@unavarra.es 